\title{
TOPOLOGICALLY 4-CHROMATIC GRAPHS AND SIGNATURES OF ODD CYCLES
}

\author{
GORD SIMONS, CLAUDE TARDIF, DAVID WEHLAU
}

\begin{abstract}
We investigate group-theoretic "signatures" of odd cycles of a graph, and their connections to topological obstructions to 3-colourability. In the case of signatures derived from free groups, we prove that the existence of an odd cycle with trivial signature is equivalent to having the coindex of the hom-complex at least 2 (which implies that the chromatic number is at least 4 ). In the case of signatures derived from elementary abelian 2-groups we prove that the existence of an odd cycle with trivial signature is a sufficient condition for having the index of the hom-complex at least 2 (which again implies that the chromatic number is at least 4 ).
\end{abstract}

Keywords: Graph colourings, homomorphisms, Hom complexes, free groups AMS 2010 Subject Classification: 05C15.

\section{INTRODUCTION}

This paper is motivated by so-called "topological bounds" on the chromatic number of a graph:

$$
\chi(H) \geq \operatorname{ind}\left(\operatorname{Hom}\left(K_{2}, H\right)\right)+2 \geq \operatorname{coind}\left(\operatorname{Hom}\left(K_{2}, H\right)\right)+2 .
$$

Here, $\operatorname{Hom}\left(K_{2}, H\right)$ is a "hom-complex" which can be viewed both as a $\mathbb{Z}_{2}$-poset and as the geometric realisation of its order complex. Its index $\operatorname{ind}\left(\operatorname{Hom}\left(K_{2}, H\right)\right)$ and coindex coind $\left(\operatorname{Hom}\left(K_{2}, H\right)\right)$ will defined in the next section.

The bounds (1) have been useful in determining chromatic numbers for various classes of graphs. However for general graphs, the index and coindex are not known to be computable. In contrast, the chromatic number is in NP. The computational aspects of such topological invariants are now being investigated (see 2,11). In this paper we focus on the case when the bounds give a chromatic number of at least 4. We present an algebraic approach.

For a graph $H$, let $A(H)$ denote the set of its arcs. That is, for $[u, v] \in E(H)$, $A(H)$ contains the two $\operatorname{arcs}(u, v)$ and $(v, u)$. Let $\mathcal{V}$ be a variety of groups (in the sense of universal algebra: a class of groups defined by a set of identities). Let $\mathcal{F}_{\mathcal{V}}(A(H))$ be the free group in $\mathcal{V}$ generated by the elements of $A(H)$. We define the congruence $\theta$ on $\mathcal{F}_{\mathcal{V}}(A(H))$ by the relations

$$
(a, b)(c, b)^{-1}(c, d)(a, d)^{-1} \quad \theta \quad 1
$$

for all 4-cycles $a, b, c, d$ of $H$. The group $\mathcal{G}_{\mathcal{V}}(H)$ is defined as the quotient $\mathcal{F}_{\mathcal{V}}(A(H)) / \theta$. Let $C_{n}$ denote the cycle with vertex-set $\mathbb{Z}_{n}=\{0, \ldots, n-1\}$ and edges $[i, i+1]$, $i \in \mathbb{Z}_{n}$. If $n$ is odd and $f: C_{n} \rightarrow H$ is a homomorphism, we define the $\mathcal{V}$-signature

The third author's research is supported by grants from NSERC and ARP. 
$\sigma_{\mathcal{V}}(f)$ of $f$ as

$$
\sigma_{\mathcal{V}}(f)=\prod_{i=0}^{n-1}(f(2 i), f(2 i+1)) \cdot(f(2 i+2), f(2 i+1))^{-1},
$$

where the indices are taken modulo $2 n+1$ and the product is developed left to right: $\prod_{i=0}^{k} x_{i}=x_{0} x_{1} \cdots x_{k}$ rather than $x_{k} x_{k-1} \cdots x_{0}$.

We will consider two varieties of groups and related signatures: the variety $\mathcal{V}_{1}$ of all groups and the variety $\mathcal{V}_{2}$ of elementary abelian 2-groups (where we use additive notation). We let $\sigma_{1}(f)$ and $\sigma_{2}(f)$ denote respectively $\sigma_{\mathcal{V}_{1}}(f)$ and $\sigma_{\mathcal{V}_{2}}(f)$. We prove the following results.

Theorem 1. Let $H$ be a graph. Then $\operatorname{coind}\left(\operatorname{Hom}\left(K_{2}, H\right)\right) \geq 2$ if and only if for some odd $n$, there exists a homomorphism $f: C_{n} \rightarrow H$ such that $\sigma_{1}(f)=1$.

Theorem 2. Let $H$ be a graph. If for some odd $n$ there exists a homomorphism $f: C_{n} \rightarrow H$ such that $\sigma_{2}(f)=0$, then $\operatorname{ind}\left(\operatorname{Hom}\left(K_{2}, H\right)\right) \geq 2$.

We will show that the existence of an odd $n$ and a homomorphism $f: C_{n} \rightarrow H$ such that $\sigma_{2}(f)=0$ can be decided in polynomial time. Therefore if the converse of Theorem 2 holds, then the question as to whether a graph $H$ satisfies $\operatorname{ind}\left(\operatorname{Hom}\left(K_{2}, H\right)\right) \geq 2$ can be decided in polynomial time. In contrast, Theorem 1 provides a necessary and sufficient algebraic condition for a graph $H$ to satisfy $\operatorname{coind}\left(\operatorname{Hom}\left(K_{2}, H\right)\right) \geq 2$, but it is not clear whether this condition can be decided at all, let alone in polynomial time. In fact, as pointed out by Zimmerman [8], it is not clear whether the word problem in $\mathcal{G}_{\mathcal{V}_{1}}(H)$ is always decidable.

\section{Preliminaries}

In this section we introduce the terminology necessary to define the index and coindex of hom-complexes, and characterise them in ways that will allow us to prove Theorems 1 and 2 ,

2.1. Topology. The hom-complex $\operatorname{Hom}\left(K_{2}, H\right)$ of $H$ is the set with elements $(A, B)$ such that $A, B$ are nonempty subsets of $H$ and every element of $A$ is joined by an edge of $H$ to every element of $B$. Here, $K_{2}$ denotes the complete graph on two vertices 0 and 1 ; the name "hom"-complex is derived from the fact that if $(A, B) \in \operatorname{Hom}\left(K_{2}, H\right)$, then for any $a \in A$ and $b \in B$, there is a homomorphism $f$ of $K_{2}$ to $H$ defined by $f(0)=a$ and $f(1)=b$.

We view $\operatorname{Hom}\left(K_{2}, H\right)$ primarily as a $\mathbb{Z}_{2}$-poset, that is a poset with a fixed-point free automorphism of order two (denoted -). The order relation on $\operatorname{Hom}\left(K_{2}, H\right)$ is coordinatewise inclusion, and the $\mathbb{Z}_{2}$ involution is given by $-(A, B)=(B, A)$. A $\mathbb{Z}_{2}$-map between $\mathbb{Z}_{2}$-posets $P$ and $Q$ is an order-preserving map $f: P \rightarrow Q$ such that $f(-x)=-f(x)$.

Any poset $P$ can also be viewed as a simplicial complex, by viewing chains as simplices. The geometric realization of $P$ is the topological space $\Delta P \subseteq \mathbb{R}^{P}$ induced by the functions $f: P \rightarrow[0,1]$ whose support (i.e., set of elements with nonzero image) is a chain in $P$, and which satisfy $\sum\{f(x): x \in P\}=1$. If $P$ is a $\mathbb{Z}_{2^{-}}$ poset, then $\Delta P$ is a $\mathbb{Z}_{2}$-space, that is, a topological space with a fixed-point free homeomorphism of order 2. A $\mathbb{Z}_{2}$-map between $\mathbb{Z}_{2}$-spaces $X$ and $Y$ is a continous map $f: X \rightarrow Y$ such that $f(-x)=-f(x)$. 
The index $\operatorname{ind}(X)$ and coindex $\operatorname{coind}(X)$ of a $\mathbb{Z}_{2}$-space $X$ are defined in terms of the unit sphere $S_{n} \subseteq \mathbb{R}^{n+1}$, viewed as a $\mathbb{Z}_{2}$-space:

- $\operatorname{ind}(X)$ is the smallest $n$ such that $X$ admits a $\mathbb{Z}_{2}$-map to $S_{n}$;

- $\operatorname{coind}(X)$ is the largest $n$ such that $S_{n}$ admits a $\mathbb{Z}_{2}$-map to $X$.

The fact that $\operatorname{ind}\left(S_{n}\right)=\operatorname{coind}\left(S_{n}\right)=n$ is not trivial, but is a restatement of the Borsuk-Ulam theorem.

For a $\mathbb{Z}_{2}$-poset $P$, we write ind $(P)$ and coind $(P)$ respectively for ind $(\Delta P)$ and $\operatorname{coind}(\Delta P)$. Now consider the $(2 n+2)$-element $\mathbb{Z}_{2}$-poset $Q_{n}$, with elements $\{ \pm 0$, $\ldots, \pm n\}$ ordered by the relation $\{+i,-i\}<\{+j,-j\}$ (in $Q_{n}$ ) when $i<j$ (in $\mathbb{N}$ ). Then $Q_{n}$ is the face-poset of the cross-polytope of dimension $n$, and therefore $\Delta Q_{n}$ is $\mathbb{Z}_{2}$-homeomorphic to $S_{n}$. This correspondence can be used to characterise the index and the coindex of a $\mathbb{Z}_{2}$-poset in terms of order-preserving $\mathbb{Z}_{2}$-maps.

The barycentric subdivision of a poset $P$ is the poset $S(P)$ whose elements are the chains of $P$, ordered by inclusion. Note that when $P$ is a $\mathbb{Z}_{2}$-poset, $S(P)$ is also a $\mathbb{Z}_{2}$-poset. The exponential notation is used to denote iterated barycentric subdivisions. By simplicial approximation, the following holds for any $\mathbb{Z}_{2}$-poset $P$ :

- $\operatorname{ind}(P)$ is the smallest $n$ such that for some $m, S^{m}(P)$ admits a $\mathbb{Z}_{2}$-map to $Q_{n}$

- $\operatorname{coind}(P)$ is the largest $n$ such that for some $m, S^{m}\left(Q_{n}\right)$ admits a $\mathbb{Z}_{2}$-map to $P$.

2.2. Graph theory. For a graph $H$, the characterisation of $\operatorname{ind}\left(\operatorname{Hom}\left(K_{2}, H\right)\right)$ given just above will be sufficient to prove Theorem 2 in Section 4 below. For a proof of Theorem 1, we rely on a further characterisation of coind $\left(\operatorname{Hom}\left(K_{2}, H\right)\right)$ in terms of graph homomorphisms.

The categorical product of two graphs $G$ and $G^{\prime}$ is the graph $G \times G^{\prime}$ defined by

$$
\begin{aligned}
V\left(G \times G^{\prime}\right) & =V(G) \times V\left(G^{\prime}\right), \\
E\left(G \times G^{\prime}\right) & =\left\{\left[\left(u, u^{\prime}\right),\left(v, v^{\prime}\right)\right]:[u, v] \in E(G) \text { and }\left[u^{\prime}, v^{\prime}\right] \in E\left(G^{\prime}\right)\right\} .
\end{aligned}
$$

For $q \in \mathbb{N}^{*}$, let $\mathbb{P}_{q}$ denote the path with vertices $0,1, \ldots, q$ linked consecutively, with a loop at 0 . For a graph $G$, the $q$-th cone $M_{q}(G)$ (or $q$-th generalised Mycielskian) over $G$ is the graph $\left(G \times \mathbb{P}_{q}\right) / \sim_{q}$, where $\sim_{q}$ is the equivalence which identifies all vertices whose second coordinate is $q$. The vertex $(V(G) \times\{q\}) / \sim_{q}$ is called the apex of $M_{q}(G)$, while $V(G) \times\{0\}$ is the base of $M_{q}(G)$. Any set $V(G) \times\{i\}$ is called a level of $M_{q}(G)$.

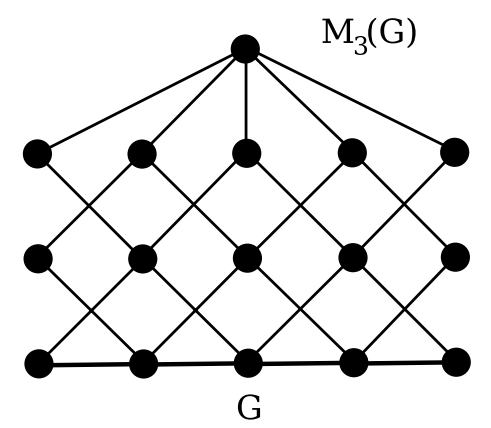

Figure 1. Generalised Mycielskian 
The cone construction allows us to define classes of "generalised Mycielski graphs" inductively: Let $\mathcal{K}_{2}=\left\{K_{2}\right\}$, and for $k \geq 3$, put

$$
\mathcal{K}_{k}=\left\{M_{q}(G): G \in \mathcal{M}_{k-1}, q \in \mathbb{N}^{*}\right\} .
$$

Lemma $3([7])$. For any graph $H$, coind $\left(\operatorname{Hom}\left(K_{2}, H\right)\right)$ is the largest $k$ such that there exist a $G \in \mathcal{K}_{k+2}$ admitting a homomorphism to $H$.

The complex used in [7] was the box complex $\mathrm{B}(H)$ rather than the hom-complex $\operatorname{Hom}\left(K_{2}, H\right)$. However the two complexes are $\mathbb{Z}_{2}$-homotopy equivalent by a result of Csorba [3].

\section{Proof of Theorem 1}

3.1. Overview of the proof. One implication of Theorem 1 has been proved in [7]:

Lemma 4 (Proposition 5 of [7]). If coind $\left(\operatorname{Hom}\left(K_{2}, H\right)\right) \geq 2$, then there exists an odd cycle $C_{n}$ and a homomorphism $f: C_{n} \rightarrow H$ such that $\sigma_{1}(f)=1$.

Proof. For reference it is worthwhile to give a sketch of the proof here. In view of Lemma 3, our hypothesis implies the existence of a homomorphism $g: M_{q}\left(C_{n}\right) \rightarrow$ $H$. We will write $u_{i, j}$ for $g\left((i, j) / \sim_{q}\right)$. (Note that $(i, j) / \sim_{q}=\{(i, j)\}$ except when $j=q ; u_{i, q}$ is the image of the apex of $M_{q}\left(C_{n}\right)$ for any $i \in \mathbb{Z}_{n}$.) We will show that the homomorphism $f: C_{n} \rightarrow H$ defined by $f(i)=u_{i, 0}$ satisfies $\sigma_{1}(f)=1$.

Consider the expressions $L_{j}, R_{j}, j=0, \ldots, q-1$ in $\mathcal{G}_{\mathcal{V}_{1}}(H)$ given by

$L_{j}=\left(u_{0, j}, u_{1, j+1}\right)\left(u_{2, j}, u_{1, j+1}\right)^{-1}\left(u_{2, j}, u_{3, j+1}\right)\left(u_{4, j}, u_{3, j+1}\right)^{-1} \cdots\left(u_{n-1, j}, u_{0, j+1}\right)$,

$R_{j}=\left(u_{0, j+1}, u_{1, j}\right)^{-1}\left(u_{2, j+1}, u_{1, j}\right)\left(u_{2, j+1}, u_{3, j}\right)^{-1}\left(u_{4, j}, u_{3, j+1}\right) \cdots\left(u_{n-1, j}, u_{0, j+1}\right)$.

We then have

$$
L_{j} R_{j}=\prod_{i=0}^{n-1}\left(u_{2 i, j}, u_{2 i+1, j+1}\right)\left(u_{2 i+2, j}, u_{2 i+1, j+1}\right)^{-1}, j=0, \ldots, q-1 .
$$

In particular, $L_{q-1} R_{q-1}$ simplifies to 1 , since $u_{2 i+1, q}$ is the constant image of the apex of $M_{q}\left(C_{n}\right)$. Also, by definition of $\theta$, for $j=1, \ldots, q-1$, we have

$$
\left(u_{2 i, j}, u_{2 i+1, j+1}\right)\left(u_{2 i+2, j}, u_{2 i+1, j+1}\right)^{-1}=\left(u_{2 i, j}, u_{2 i+1, j-1}\right)\left(u_{2 i+2, j}, u_{2 i+1, j-1}\right)^{-1}
$$

for $i=0, \ldots, n-1$. Therefore

$$
L_{j} R_{j}=\prod_{i=0}^{n-1}\left(u_{2 i, j}, u_{2 i+1, j-1}\right)\left(u_{2 i+2, j}, u_{2 i+1, j-1}\right)^{-1} j=0, \ldots, q-1 .
$$

This is the image $\phi\left(R_{j-1} L_{j-1}\right)$ of $R_{j-1} L_{j-1}$ under the (well-defined) automorphism $\phi$ of $\mathcal{G}_{\mathcal{V}_{1}}(H)$ which interchanges $(x, y)$ with $(x, y)^{-1}$ for all $(x, y) \in A(H)$. Therefore if $L_{j} R_{j}=1$, then $R_{j-1} L_{j-1}=1$ and $L_{j-1} R_{j-1}=1$, since $L_{j-1} R_{j-1}$ and $R_{j-1} L_{j-1}$ are conjugates. Therefore, $L_{j} R_{j}=1$ for all $j=0, \ldots, q-1$. Again by definition of $\theta$, we then have

$$
\begin{aligned}
\sigma_{1}(f) & =\prod_{i=0}^{n-1}\left(u_{2 i, 0}, u_{2 i+1,0}\right)\left(u_{2 i+2,0}, u_{2 i+1,0}\right)^{-1} \\
& =\prod_{i=0}^{n-1}\left(u_{2 i, 0}, u_{2 i+1,1}\right)\left(u_{2 i+2,0}, u_{2 i+1,1}\right)^{-1}=L_{0} R_{0}=1 .
\end{aligned}
$$


It would be nice to be able to reverse the arguments of this proof to prove the second direction. That is, start with $f: C_{n} \rightarrow H$ such that $\sigma_{1}(f)=1$, and use the definition of $\theta$ to extend it to a homomorphism $g: M_{q}\left(C_{n}\right) \rightarrow H$. However an example in [7] shows that this is not always possible; $C_{n}$ may be to small as a base.

Thus we need to start from the basic information provided by the equation $\sigma_{1}(f)=1$. Since $\mathcal{G}_{\mathcal{V}_{1}}(H)=\mathcal{F}_{\mathcal{V}}(A(H)) / \theta$ this means that in $\mathcal{F}_{\mathcal{V}_{1}}, \sigma_{1}(f)$ is equal to a product $\prod_{i=1}^{k} \gamma_{i}^{-1} \rho_{i} \gamma_{i}$ of conjugates of the relations $\rho_{i}$ defining $\theta$.

The first difficulty here is that there is a useful feature common to $\sigma_{1}(f)$ and the generators $\rho_{i}$ of $\theta$, which is lost in the expression $\prod_{i=1}^{k} \gamma_{i} \rho_{i} \gamma_{i}^{-1}$ : the fact that words alternate in forward arcs and inverses of backward arcs along a walk in $H$. This feature provides a natural connection between algebraic expressions and path homomorphisms. For this reason, we will fix a root vertex $r$ in $H$, and associate to each arc $(x, y)$ a closed walk from $r$ through $(x, y)$, called a "loop". The use of loops will transform $\prod_{i=1}^{k} \gamma_{i} \rho_{i} \gamma_{i}^{-1}$ into a word which is much longer, but which has the desired alternating property. We will identify this word with an expression of the type $L_{j} R_{j}$ defining homomorphic images of two consecutive levels of some $M_{q}\left(C_{2 m+1}\right)$.

We will then extend this homomorphism to the apex of $M_{q}\left(C_{2 m+1}\right)$ using the definition of $\theta$, and towards its base using the simplification of $\prod_{i=1}^{k} \gamma_{i} \rho_{i} \gamma_{i}^{-1}$ to $\sigma_{1}(f)$. Our basic tool to convert algebraic simplifications to homomorphism extensions is the extension along "bricks", that is, essentially rectangular pieces that dissect $M_{q}\left(C_{2 m+1}\right)$.

The last phase of the extension will be the connection to the base of $M_{q}\left(C_{2 m+1}\right)$, which is equivalent to finding an extension that is equal on two consecutive levels.

3.2. Loops. Most of our work will be done in the free monoid $\left(A(H) \cup A(H)^{-1}\right)^{*}$ generated by $A(H) \cup A(H)^{-1}$, where $A(H)^{-1}=\left\{(u, v)^{-1}:(u, v) \in A(H)\right\}$ is a set of symbols disjoint from $A(H)$. Of course, $\mathcal{F}_{\mathcal{V}_{1}}(A(H))=\left(A(H) \cup A(H)^{-1}\right)^{*} / \iota$, where $\iota$ is the congruence which identifies $(u, v)(u, v)^{-1}$ and $(u, v)^{-1}(u, v)$ to 1 for all $(u, v) \in A(H)$. However, for a suitable correspondence between words and walks, it is sometimes useful to avoid this identification.

A walk $u_{0}, u_{1}, \ldots, u_{n}$ in $H$ is the image of a homomorphism $f$ of some path with vertices $0,1, \ldots n$ linked consecutively. To such a walk we can associate a word

$$
\omega(f)=\left(u_{0}, u_{1}\right)\left(u_{2}, u_{1}\right)^{-1}\left(u_{2}, u_{3}\right)\left(u_{4}, u_{3}\right)^{-1} \ldots
$$

ending in $\left(u_{n-1}, u_{n}\right)$ or $\left(u_{n}, u_{n-1}\right)^{-1}$ depending on whether $n$ is odd or even. This word alternates symbols from $A(H)$ and symbols from $A(H)^{-1}$, with the symbol following $\left(u_{2 i}, u_{2 i+1}\right)$ being $\left(u_{2 i+2}, u_{2 i+1}\right)^{-1}$ for some $u_{2 i+2} \in V(H)$, and the symbol following $\left(u_{2 i}, u_{2 i-1}\right)^{-1}$ being $\left(u_{2 i}, u_{2 i+1}\right)$ for some $u_{2 i+1} \in V(H)$. Conversely, a word with these properties naturally corresponds to a walk in $H$.

Now for our purposes we can assume that $H$ is connected and nonbipartite, if necessary by restricting our attention to the component of $H$ that contains an odd cycle with trivial signature. We fix a root vertex $r$ and for every vertex $u$ of $H$, we fix an even path $p_{e}(u)$ and an odd path $p_{o}(u)$, both from $r$ to $u$. For an $\operatorname{arc}(u, v)$ of $H$, we define the loop $L(u, v) \in\left(A(H) \cup A(H)^{-1}\right)^{*}$ corresponding to $(u, v)$ by

$$
L(u, v)=\omega\left(p_{e}(u)\right) \cdot(u, v) \cdot \omega\left(p_{o}(v)\right)^{-1} .
$$


Of course, $\left(a_{1} a_{2} \cdots a_{n}\right)^{-1}$ means $a_{n}^{-1} a_{n-1}^{-1} \cdots a_{1}^{-1}$, though this needs to be stated formally since inversion does not exist in $\left(A(H) \cup A(H)^{-1}\right)^{*}$. With this notation, we define $L\left((u, v)^{-1}\right)$ as $(L(u, v))^{-1}$.

The loop function naturally extends to $\left(A(H) \cup A(H)^{-1}\right)^{*}$ by putting $L\left(a_{1} \cdots a_{n}\right)=$ $L\left(a_{1}\right) \cdots L\left(a_{n}\right)$. The map $L:\left(A(H) \cup A(H)^{-1}\right)^{*} \rightarrow\left(A(H) \cup A(H)^{-1}\right)^{*}$ is an endomorphism whose image consists of words corresponding to even closed walks rooted at $r$.

3.3. Cycles with trivial signature. Now let $C_{n^{\prime}}$ be an odd cycle and $f: C_{n^{\prime}} \rightarrow$ $H$ a homomorphism such that $\sigma_{1}(f)=1$. For our purposes, it is useful to assume that for $i \in \mathbb{Z}_{n^{\prime}}=V\left(C_{n^{\prime}}\right)$, we have $f(i-1) \neq f(i+1)$. This can be done without loss of generality, since if $f(i-1)=f(i+1)$, then we can remove $i$ and identify $i-1$ and $i+1$ to create a copy of $C_{n^{\prime}-2}$ on which $f$ induces a homomorphism $f^{\prime}: C_{n^{\prime}-2} \rightarrow H$. We then have $\sigma_{1}\left(f^{\prime}\right)=\sigma_{1}(f)$, since $\sigma_{1}\left(f^{\prime}\right)$ is obtained from $\sigma_{1}(f)$ by cancelling out $(f(i-1), f(i))$ with $(f(i+1), f(i))^{-1}$ and $(f(i), f(i-1))^{-1}$ with $(f(i), f(i+1))$ (unless $i=0$, in which case we get a conjugation of the identity element instead of the second cancellation).

We begin by lifting the condition $\sigma_{1}(f)=1$ from $\mathcal{G}(H)$ to $\mathcal{F}_{\mathcal{V}_{1}}(A(H))$ : by definition, this means that there exists a sequence

$$
\left\{\rho_{i}=\left(a_{i}, b_{i}\right)\left(c_{i}, b_{i}\right)^{-1}\left(c_{i}, d_{i}\right)\left(a_{i}, d_{i}\right)^{-1}: i=1, \ldots, k\right\}
$$

of generators of $\theta$ and a sequence $\gamma_{1}, \ldots, \gamma_{k}$ of conjugating elements such that

$$
\prod_{i=0}^{n^{\prime}-1}(f(2 i), f(2 i+1)) \cdot(f(2 i+2), f(2 i+1))^{-1}=\prod_{i=1}^{k} \gamma_{i} \cdot \rho_{i} \cdot \gamma_{i}^{-1}
$$

Note that the left side is reduced in $\mathcal{F}_{\mathcal{V}_{1}}(A(H))$, since $f(j-1) \neq f(j+1)$ for all $j \in \mathbb{Z}_{n^{\prime}}$. This means that the right side simplifies to the left, by repeatedly cancelling out adjacent terms that are inverse of each other.

We view the terms in this equation as elements of $\left(A(H) \cup A(H)^{-1}\right)^{*}$ (that is, as their simplest preimage under $\iota)$. In this way the terms $L\left(\rho_{i}\right)$ and $L\left(\gamma_{i}\right)$ are well defined. Now consider the word

$$
w^{\prime}=\prod_{i=1}^{k} L\left(\gamma_{i}\right) \cdot L\left(\rho_{i}\right) \cdot L\left(\gamma_{i}^{-1}\right)
$$

Its length is a multiple of 4 , since for each $i$, the length of $L\left(\gamma_{i}\right) \cdot L\left(\rho_{i}\right) \cdot L\left(\gamma_{i}^{-1}\right)$ is a multiple of 4 . Therefore for any neighbour $s$ of $r$, the word $w=w^{\prime} \cdot(r, s) \cdot(r, s)^{-1}$ has length equal to $2 n$ for some odd $n$. We then have $w=\omega(g)$ for some closed walk $g$. We identify the domain of $g$ with the $2 n$-cycle $C_{n} \times K_{2}$, whose vertices correspond to two consecutive levels of some $M_{q}\left(C_{n}\right)$. We will show such that it is possible to choose $q$ and the correspondence such that $g$ extends to a homorphism of $M_{q}\left(C_{n}\right)$ to $H$. 
3.4. Bricks and simplifications. For integers $i$ and $j$, the bricks $B_{\mathrm{S}}(i, j)$ and $B_{\mathrm{d}}(i, j)(i, j)$ are the graphs defined by

$$
\begin{aligned}
V\left(B_{\mathrm{S}}(i, j)\right) & =\{(x, y): 0 \leq x \leq i, 0 \leq y \leq j, x \text { and } y \text { have the same parity }\} \\
E\left(B_{\mathrm{S}}(i, j)\right) & =\left\{\left[(x, y),\left(x^{\prime}, y^{\prime}\right)\right]:\left|x-x^{\prime}\right|=1,\left|y-y^{\prime}\right|=1\right\} \\
V\left(B_{\mathrm{d}}(i, j)\right) & =\{(x, y): 0 \leq x \leq i, 0 \leq y \leq j, x \text { and } y \text { have different parities }\} \\
E\left(B_{\mathrm{d}}(i, j)\right) & =\left\{\left[(x, y),\left(x^{\prime}, y^{\prime}\right)\right]:\left|x-x^{\prime}\right|=1,\left|y-y^{\prime}\right|=1\right\}
\end{aligned}
$$

Thus, $B_{\mathrm{S}}(i, j)$ and $B_{\mathrm{d}}(i, j)$ are the two connected components of the categorical product of paths of lengths $i$ and $j$. The sets of vertices of $B_{\mathrm{S}}(i, j)$ and $B_{\mathrm{d}}(i, j)$ with second coordinate 0 are called the lower side of $B_{\mathrm{S}}(i, j)$ and $B_{\mathrm{d}}(i, j)$, and similarly, their upper, left, and right sides are defined by obvious conditions.

For any $i, B_{\mathrm{S}}(2 i, 1)$ and $B_{\mathrm{d}}(2 i, 1)$ are paths of length $2 i$. We use the following extension properties of their homomorphisms to $H$.

Lemma 5. Let $h: B_{s}(2 i, 1) \rightarrow V(H), h^{\prime}: B_{s}(2 i, 1) \rightarrow V(H)$ be homomorphisms.

(i) For any $j \geq 0$, the map $\hat{h}$ defined by $\hat{h}(x, y)=h(x,(y \bmod 2))$ is a homomorphism of $B_{s}(2 i, j)$ to $H$. Similarly, for any $j \geq 0$, the map $\hat{h}^{\prime}$ defined by $\hat{h}^{\prime}(x, y)=h(x, 1-(y \bmod 2))$ is a homomorphism of $B_{d}(2 i, j)$ to $H$.

(ii) If $h(x, x \bmod 2)=h(2 i-x, x \bmod 2)$ for $x=0, \ldots i$, then there exists a homomorphism $\hat{h}: B_{s}(2 i, 2\lceil i / 2\rceil) \rightarrow H$ extending $h$ such that $\hat{h}$ is identically equal to $h(0)$ on the left, upper and right sides of $B_{s}(2 i, 2\lceil i / 2\rceil)$. Similarly, if $h^{\prime}(x, 1-(x \bmod 2))=h^{\prime}(2 i-x, 1-(x \bmod 2))$ for $x=0, \ldots i$, then there exists a homomorphism $\hat{h}^{\prime}: B_{d}(2 i, 2\lfloor i / 2\rfloor+1) \rightarrow H$ extending $h$ such that $\hat{h}$ is identically equal to $h(0,1)$ on the left, upper and right sides of $B_{d}(2 i, 2\lfloor i / 2\rfloor+1)$.

Proof. Item (i) is straightforward. To prove the first part of item (ii), we note that the distance between two vertices $(x, y),\left(x^{\prime}, y^{\prime}\right)$ of $B_{\mathrm{S}}(2 i, j)$ is the "bus distance" $\max \left\{\left|x-x^{\prime}\right|,\left|y-y^{\prime}\right|\right\}$. Therefore the map $\hat{h}: B_{\mathrm{S}}(2 i, 2\lceil i / 2\rceil) \rightarrow H$ defined by $\hat{h}(x, y)=h\left(x^{\prime}, y^{\prime}\right)$, where $\left(x^{\prime}, y^{\prime}\right) \in B_{\mathrm{S}}(2 i, 1)$ is at the same distance as $(x, y)$ from $(i,(i \bmod 2))$, is a homomorphism with the prescribed properties. The second part is proved similarly.

3.5. Extension to the apex. We apply Lemma 5 to extend the homomorphism $g: C_{n} \times K_{2} \rightarrow H$ towards the apex of some $M_{q}\left(C_{n}\right)$. Consider the restrictions $g_{1}, \ldots, g_{k}$ of $g$ such that $\omega\left(g_{i}\right)=L\left(\gamma_{i}\right) \cdot L\left(\rho_{i}\right) \cdot L\left(\gamma_{i}^{-1}\right)$. For a fixed $i$, we further decompose $g_{i}$ into five restrictions $h_{a}, h_{b}, h_{c}, h_{d}, h_{a}^{\prime}$ such that

$$
\begin{aligned}
\omega\left(h_{a}\right) & =L\left(\gamma_{i}\right) \cdot \omega\left(p_{e}\left(a_{i}\right)\right), \\
\omega\left(h_{b}\right) & =\omega\left(p_{o}\left(b_{i}\right)\right)^{-1} \circ \omega\left(p_{o}\left(b_{i}\right)\right), \\
\omega\left(h_{c}\right) & =\omega\left(p_{e}\left(c_{i}\right)\right)^{-1} \circ \omega\left(p_{e}\left(c_{i}\right)\right), \\
\omega\left(h_{d}\right) & =\omega\left(p_{o}\left(d_{i}\right)\right)^{-1} \circ \omega\left(p_{o}\left(d_{i}\right)\right), \\
\omega\left(h_{a}^{\prime}\right) & =\omega\left(p_{e}\left(a_{i}\right)\right)^{-1} \cdot L\left(\gamma_{i}\right) .
\end{aligned}
$$

By Lemma 5 (ii), $h_{c}$ extends to a homomorphism $\hat{h}_{c}$ of some $B_{c}=B_{\mathrm{S}}(2 \ell, \ell)$ to $H$ ( $\ell$ being the length of $p_{e}\left(c_{i}\right)$ ), which is identically equal to $c_{i}$ on its left, upper and right sides. Similarly $h_{b}$ and $h_{d}$ extend to $\hat{h}_{b}$ and $\hat{h}_{d}$, which are identically 


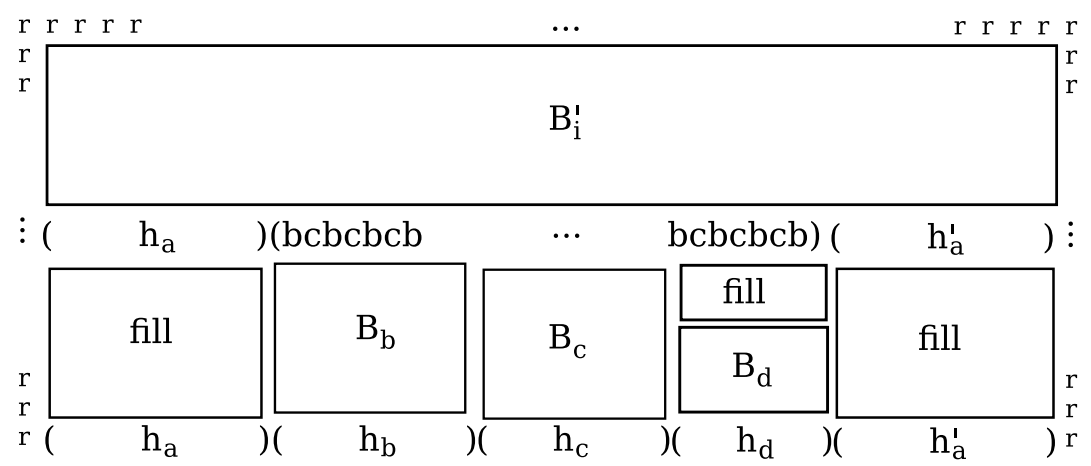

Figure 2. Extension of $g_{i}$

equal to $b_{i}$ and $d_{i}$ respectively on their left, upper and right sides. Moreover, the extensions $\hat{h}_{b}, \hat{h}_{c}, \hat{h}_{d}$ of $h_{b}, h_{c}, h_{d}$ to $B_{b}, B_{c}, B_{d}$ can be carried simultaneously side by side, since $c_{i}$ is adjacent to $b_{i}$ and $d_{i}$.

We then add one level to $B_{c}$ and extend $\hat{h}_{c}$ by mapping the new level to $b_{i}$, and add two levels to each of $B_{b}$ and $B_{d}$ and extend $\hat{h}_{b}$ and $\hat{h}_{d}$ by mapping the new levels to $c_{i}$ and $b_{i}$ respectively. We then use Lemma 5 (i) on the top two levels of $Q_{b}, Q_{c}$ and $Q_{d}$ to add levels and equalize their heights if necessary. We can then use Lemma 5 (i) to extend $h_{a}$ and $h_{a}^{\prime}$ upwards to match the height of $B_{b}, B_{c}$ and $B_{d}$.

We have thus extended $g_{i}$ to $\hat{g}_{i}: B_{i} \rightarrow H$ such that the restriction $g_{i}^{\prime}$ of $\hat{g}_{i}$ to the top two levels of $Q_{i}$ satisfies

$\omega\left(g_{i}^{\prime}\right)=L\left(\gamma_{i}\right) \circ \omega\left(p_{e}\left(a_{i}\right)\right) \circ\left(a_{i}, b_{i}\right)\left(\left(c_{i}, b_{i}\right)^{-1}\left(c_{i}, b_{i}\right)\right)^{e_{i}}\left(a_{i}, b_{i}\right)^{-1} \circ \omega\left(p_{e}\left(a_{i}\right)\right)^{-1} \circ L\left(\gamma_{i}\right)^{-1}$,

for some $e_{i}$ (see Figure 2). Using Lemma 5 (ii), we extend $g_{i}^{\prime}$ to $\hat{g}_{i}^{\prime}: B_{i}^{\prime} \rightarrow H$ which is identically $r$ on the left, top and right sides of $B_{i}^{\prime}$.

These extensions of $g_{i}$ to $\hat{g}_{i}$ and $\hat{g}_{i}^{\prime}$ can be carried simultaneously for $i=1, \ldots, k$, since they all have the value $r$ at their common boundaries. We can then use Lemma 5 (i) to equalize heights, and bring up the part of $g$ corresponding to $(r, s)(r, s)^{-1}$ to the same height. Since the value of the extensions is identically $r$ at the top level, we can identify all the vertices of this top level. We have extended $g$ to the apex of some $M_{q}\left(C_{n}\right)$.

3.6. Extension towards the base. The extension of $g$ towards the base of $M_{q}\left(C_{n}\right)$ is a second extension independent from the extension to the apex. It again uses Lemma 5, thus we keep the terminology of extending in the "upper" direction. The two extensions will afterwards be merged together by identifying their bottom level.

In $\mathcal{F}_{\mathcal{V}_{1}}(A(H))$, the word $\prod_{i=1}^{k} \gamma_{i} \cdot \rho_{i} \cdot \gamma_{i}^{-1}$ of $\left(A(H) \cup A(H)^{-1}\right)^{*}$ simplifies to

$$
\sigma_{1}(f)=\prod_{i=0}^{n^{\prime}-1}(f(2 i), f(2 i+1)) \cdot(f(2 i+2), f(2 i+1))^{-1} .
$$

Specifically, this means that there exists a sequence of basic simplifications of $\prod_{i=1}^{k} \gamma_{i} \cdot \rho_{i} \cdot \gamma_{i}^{-1}$ eliminating everything but the terms of $\sigma_{1}(f)$. In each of these basic simplification, some term $t_{i}=x$ will cancel out either with the next term 
$t_{i+1}=x^{-1}$, or with a further term $t_{i+2 j}=x^{-1}$, where all the intermediate terms $t_{i+1}, \ldots, t_{i+2 j-1}$ have been previously simplified.

In $\prod_{i=1}^{k} L\left(\gamma_{i} \cdot \rho_{i} \cdot \gamma_{i}^{-1}\right)$, a term $t_{i}$ is replaced by $L\left(t_{i}\right)$. Consider the homomorphism corresponding to the word $L\left(t_{i}\right) \circ\left((r, s)(r, s)^{-1}\right)^{e_{i}} \circ L\left(t_{i}^{-1}\right)$ for some $e_{i}$. By Lemma 5 (ii), it extends to a homomorphism of some $B_{i}$ to $H$ with value identically $r$ on its left, upper and right sides. We can then add a level with value identically $s$, and use Lemma 5 (i) to bring up the remainder of the extension of $g$ to the same level.

In this way, we match each step in the simplification of $\prod_{i=1}^{k} \gamma_{i} \cdot \rho_{i} \cdot \gamma_{i}^{-1}$ to a corresponding step in the extension. We end up with an extension where the two upper levels correspond to a word with the terms $L(f(2 i), f(2 i+1))$ and $L(f(2 i+$ $2), f(2 i+1))^{-1}$ separated by terms of the form $\left((r, s)(r, s)^{-1}\right)^{e}$.

Now in this word, between $(f(2 i), f(2 i-1))^{-1}$ and $(f(2 i), f(2 i+1))$ is a word of the form $\omega\left(p_{e}(f(2 i))^{-1} \circ\left((r, s)(r, s)^{-1}\right)^{e} \circ \omega\left(p_{e}(f(2 i))\right.\right.$. By Lemma 5 (ii), it extends to a homomorphism of some brick to $H$ with value identically $f(2 i)$ on its left, upper and right sides. We can then add a level with value identically $f(2 i-1)$. Similarly between $(f(2 i), f(2 i+1))$ and $(f(2 i+2), f(2 i+1))^{-1}$ is a word of the form $\omega\left(p_{o}(f(2 i+1))^{-1} \circ\left((r, s)(r, s)^{-1}\right)^{e} \circ \omega\left(p_{o}(f(2 i+1))\right.\right.$. By Lemma 5 (ii), it extends to a homomorphism of some brick to $H$ with value identically $f(2 i+1)$ on its left, upper and right sides. We can then add a level with value identically $f(2 i)$.

These extensions can be carried out side by side simultaneously, since $f(j)$ is adjacent to $f(j+1)$ for all $j$. We use Lemma 5 (i) to equalize the height. We now have extended $g$ so that (after a suitable cyclic shift), the homomorphism on the top two levels corresponds to a word of the form

$$
\begin{aligned}
& \prod_{i=0}^{n^{\prime}-1}\left[\left((f(2 i), f(2 i+1))(f(2 i), f(2 i+1))^{-1}\right)^{e_{i}}(f(2 i), f(2 i+1))\right. \\
& \left.\cdot\left((f(2 i+2), f(2 i+1))^{-1}(f(2 i+2), f(2 i+1))\right)^{e_{i}^{\prime}}(f(2 i+2), f(2 i+1))^{-1}\right] .
\end{aligned}
$$

3.7. Connection to the base. Let $f^{\prime}: C_{n} \times K_{2} \rightarrow H$ be the homomorphism corresponding to the top two levels of the second extension of $g$. Note that $f^{\prime}$ follows the original $f$ twice around its image, with every arc traced back and forth many times. More precisely, $f^{\prime}=h \circ f$, where $h: C_{n} \times K_{2} \rightarrow C_{n^{\prime}}$ is a homomorphism. The only thing missing is to have $h(i, 0)=h(i, 1)$ for all $i$, that is, to have $f^{\prime}$ equal on the two levels. These levels could then be identified to form the base of $M_{q}\left(C_{n}\right)$.

We label the vertices on $C_{n} \times K_{2}=C_{2 n}$ consecutively $u_{0}, \ldots, u_{2 n-1}$ (with indices in $\left.\mathbb{Z}_{2 n}\right)$. We label the edge $\left[u_{i}, u_{i+1}\right]$ with the sign $+($ resp. -$)$ if for some $j \in \mathbb{Z}_{n^{\prime}}$ we have $h\left(u_{i}\right)=j$ and $h\left(u_{i+1}\right)=j+1\left(\operatorname{resp} h\left(u_{i+1}\right)=j-1\right)$. The word $\omega\left(f^{\prime}\right)$ above shows that the number of edges with label + is $n+n^{\prime}$, and the number of edges with label - is $n-n^{\prime}$.

Opposite signs on two consecutive edges $\left[u_{i}, u_{i+1}\right],\left[u_{i+1}, u_{i+2}\right]$ happen precisely when $h\left(u_{i}\right)=h\left(u_{i+2}\right)$. We then have $h\left(u_{i+1}\right) \in\left\{h\left(u_{i}\right)-1, h\left(u_{i}\right)+1\right\}$. Substituting one value for the other will interchange the signs of $\left[u_{i}, u_{i+1}\right]$ and $\left[u_{i+1}, u_{i+2}\right]$. In terms of the extension of $g$, this corresponds to adding two levels to match the operation. That is, the extension on the two new levels is identical to the extension on the previous two levels, except that value at the vertex corresponding to $u_{i+1}$ switches from one term in $\left\{f\left(h\left(u_{i}\right)-1\right), f\left(h\left(u_{i}\right)+1\right)\right\}$ to the other.

Proceeding this way, we can move the labels around in any way we please. In particular we can rearrange the labels until there are $\left(n+n^{\prime}\right) / 2$ "+" labels followed by $\left(n-n^{\prime}\right) / 2$ " -" labels, then by $\left(n+n^{\prime}\right) / 2$ "+" labels and $\left(n-n^{\prime}\right) / 2$ "-" labels. 
In this way, between any $u_{i}$ and $u_{i+n}$, the value of $h$ moves forward $\left(n+n^{\prime}\right) / 2$ times and backward $\left(n-n^{\prime}\right) / 2$ times so that it ends up $n^{\prime}$ places forward in $C_{n^{\prime}}$, exactly where it started. Our second extension of $g$ is then equal on the top two levels, so that these levels can be identified to form the base of $M_{q}\left(C_{n}\right)$. This concludes the proof of Theorem 1

\section{Proof of Theorem 2}

4.1. Algorithmic considerations. To prove Theorem 2. we will show that the existence of an odd cycle in $H$ with zero signature is incompatible with the existence of a $\mathbb{Z}_{2}$-map of some $S^{m}\left(\operatorname{Hom}\left(K_{2}, H\right)\right)$ to $Q_{1}$. Note that Hom and $S$ are both exponential constructions. We will first show that the detection of an odd cycle with zero signature can be done efficiently in terms of the size of $H$.

To each $\operatorname{arc}(u, v)$ of $H$ we associate a variable $X_{u, v} \in \mathbb{Z}_{2}$. We consider the system consting of the following equations.

- The flow constraint at a vertex $u$ of $H$ is the equation

$$
\sum_{v \in N_{H}(u)}\left(X_{u, v}+X_{v, u}\right)=0 .
$$

(Where $N_{H}(u)$ is the neighbourhood of $u$ in $H$.)

- The parity constraint is the global condition

$$
\sum_{(u, v) \in A(H)} X_{u, v}=1 .
$$

- The signature constraint is the equation

$$
\sum_{(u, v) \in A(H)}\left(\left(X_{u, v}-X_{v, u}\right) \cdot(u, v)\right)=0 .
$$

The flow and parity constraints are equations in $\mathbb{Z}_{2}$. The signature constraint is a single equation in $\mathcal{G}_{\mathcal{V}_{2}}(H)$. The group $\mathcal{G}_{\mathcal{V}_{2}}(H)$ has a minimal generating set with no more than $|A(H)|$ elements. Each $\operatorname{arc}(u, v)$ can be expressed in terms of this generating set. The signature constraint is then the set of constraints corresponding to voiding the coefficient of each element of this generating set in the expression $\sum_{(u, v) \in A(H)}\left(X_{u, v}-X_{v, u}\right) \cdot(u, v)$. Thus the system has no more than $|V(H)|+1+$ $|A(H)|$ linear equations in $|A(H)|$ variables.

If for some odd cycle $C_{n}$ there is a homomorphism $f: C_{n} \rightarrow H$ such that $\sigma_{2}(f)=0$, then the system above has a solution, obtained by putting $X_{u, v}=1$ if there is an odd number of elements $i$ of $\mathbb{Z}_{n}$ such that $f(i)=u$ and $f(i+1)=v$, and $X_{u, v}=0$ otherwise. Conversely, for each solution of the system, the subdigraph of $H$ spanned by the $\operatorname{arcs}(u, v)$ such that $X_{u, v}=1$ is Eulerian, though not necessarily connected. We can modify the solution by giving the value 1 to variables $X_{u, v}$, $X_{v, u}$ corresponding to opposite arcs, to make the subdigraph connected. Indeed it is clear that this modification does not alter the validity of the solution. An Euler tour of the subdigraph then corresponds to a homomorphism $f: C_{n} \rightarrow H$ with $n$ odd, such that $\sigma_{2}(f)=0$. Thus, the existence of some odd cycle with zero signature can be detected in polynomial time. 
4.2. $\mathbb{Z}_{2}$-maps of crown. Most of our work will involve groups of the form $\mathbb{Z}_{2}^{P^{2}}$, where $P$ is a poset. Here, $\underline{2}$ is the poset with elements 0,1 such that $0<1$. For a poset $P, P^{2}$ is the set of all order-preserving maps of $\underline{2}$ to $P$, including the constant maps. We will represent an element of $P^{2}$ by the comparability $(x \leq y)$ it represents. $\mathbb{Z}_{2}^{P^{2}}$ is the 2 -group generated by $P^{2}$. To an order preserving-map $f: P \rightarrow Q$, we naturally associate the group homomorphism $\hat{f}: \mathbb{Z}_{2}^{P^{\underline{2}}} \rightarrow \mathbb{Z}_{2}^{Q^{\underline{2}}}$ which extends the map between generators defined by $f$.

A $\mathbb{Z}_{2}$-crown is a $\mathbb{Z}_{2}$-poset $P$ with elements $\pm 0, \ldots, \pm(2 n-1)$ and the relations

$0<1>2<3>\cdots<2 n-1>-0<-1>-2<-3>\cdots<-(2 n-1)>0$.

The strict order indicator $1_{P}^{<}$on $P$ is the element of $\mathbb{Z}_{2}^{P}{ }^{2}$ with value 1 on injective maps and 0 on constant maps. Our argument is partly based on the following result, which is a simplicial statement of the fact that an antipodal continuous self-map of the circle has "odd degree".

Lemma 6. Let $P$ be a $\mathbb{Z}_{2}$-crown and $f: P \rightarrow Q_{1}$ a $\mathbb{Z}_{2}$-map. Then $\hat{f}\left(1_{P}^{<}\right)=1_{Q_{1}}^{<}$.

Proof. Each connected component of $f^{-1}(+0)$ and of $f^{-1}(-0)$ starts and ends in an even number, and each connected component of $f^{-1}(+1)$ and of $f^{-1}(-1)$ starts and ends in an odd number. Thus the coefficient of each of $(+0,+0),(-0,-0)$, $(+1,+1),(-1,-1)$ in $\hat{f}\left(1_{P}^{<}\right)$is 0 . Now suppose without loss of generality that $f(0)=0$ and $f(-0)=-0$. Then on the "positive zig-zag" $0<1>2<3>$ $\cdots<2 n-1>-0$, the image of $f$ will switch from +0 to -0 some $n$ times, and switch back from -0 to $+0 n-1$ times. Every switch goes through +1 or -1 , so one of the pairs $(+0,+1),(-0,+1)$ or $(+0,-1),(-0,-1)$ of strict comparibilities is touched an odd number of times, and the other an even number of times. Since $f$ is a $\mathbb{Z}_{2}$-map, these numbers are reversed on the "negative zig-zag" $-0<-1>-2<-3>\cdots<-2 n+1>0$, so that each of the strict comparibilities $(+0,+1),(-0,+1),(+0,-1),(-0,-1)$ is touched an odd number of times. Thus $\hat{f}\left(1_{P}^{<}\right)=1_{Q_{1}}^{<}$.

4.3. Cycles with null signature. A graph homomorphism $g: C_{n} \rightarrow H$ induces the $\mathbb{Z}_{2}$-map $g^{\prime}: \operatorname{Hom}\left(K_{2}, C_{n}\right) \rightarrow \operatorname{Hom}\left(K_{2}, H\right)$ defined by $g^{\prime}(A, B)=(g(A), g(B))$. However, it will be useful to associate to $g$ a different map $g^{+}: \operatorname{Hom}\left(K_{2}, C\right) \rightarrow$ $\operatorname{Hom}\left(K_{2}, H\right)$ defined as follows.

- A minimal element $(\{i\},\{j\})$ of $\operatorname{Hom}\left(K_{2}, C_{n}\right)$ correspond to an arc $(i, j)=$ $(i, i \pm 1)$ of $C$, and we put $g^{+}(\{i\},\{j\})=(\{f(i)\},\{f(j)\})$, its natural image induced by $g$.

- For a maximal element of the form $(\{i\},\{i-1, i+1\})$, we put $g^{+}(\{i\},\{i-$ $1, i+1\})=\left(\{g(i)\}, N_{H}(g(i))\right)$. Similarly, for a maximal element of the form $(\{i-1, i+1\},\{i\})$, we put $g^{+}(\{i-1, i+1\},\{i\})=\left(N_{H}(g(i)),\{g(i)\}\right)$.

Thus if $f: \operatorname{Hom}\left(K_{2}, H\right) \rightarrow Q_{1}$ is a $\mathbb{Z}_{2}$-map, then $\widehat{f \circ g^{+}}\left(1_{\operatorname{Hom}\left(K_{2}, C\right)}^{<}\right)=1_{Q_{1}}^{<}$by Lemma 6. The same holds with barycentric subdivisions: $S^{m}\left(\operatorname{Hom}\left(K_{2}, C\right)\right)$ is a $\mathbb{Z}_{2^{-}}$ crown, and if $f: S^{m}\left(\operatorname{Hom}\left(K_{2}, H\right)\right) \rightarrow Q_{1}$ is a $\mathbb{Z}_{2}$-map, then $\left.f \circ \widehat{S^{m}\left(g^{+}\right.}\right)\left(1_{S^{m}\left(\operatorname{Hom}\left(K_{2}, C\right)\right)}^{<}\right)=$ $1_{Q_{1}}^{<}$

Now, if $\sigma_{2}(g)=0$, then in $\mathcal{F}_{\mathcal{V}_{2}}(A(H))$ we have

$$
\sigma_{2}(g)=\sum_{i=0}^{n-1}[(g(2 i), g(2 i+1))+(g(2 i+2), g(2 i+1))]=\sum_{j=1}^{k} \rho_{j},
$$


G. SIMONS, C. TARDIF, D. WEHLAU

where $\rho_{1}, \ldots, \rho_{k}$ are relations of the form $\rho_{j}=\left(a_{j}, b_{j}\right)+\left(c_{j}, b_{j}\right)+\left(c_{j}, d_{j}\right)+\left(a_{j}, d_{j}\right)$ defining the congruence $\theta$ on $\mathcal{F}_{\mathcal{V}_{2}}(A(H))$. To $\rho=(a, b)+(c, b)+(c, d)+(a, d)$, we associate the subposet $\rho^{+}$of $\operatorname{Hom}\left(K_{2}, H\right)$ induced by the the set $\left\{\left(\{a\}, N_{H}(a)\right)\right.$, $\left.\left(N_{H}(b),\{b\}\right),\left(\{c\}, N_{H}(c)\right),\left(N_{H}(d),\{d\}\right),(\{a\},\{b\}),(\{c\},\{b\}),(\{c\},\{d\}),(\{a\},\{d\})\right\}$. (See Figure 3.)

Our next Lemma adapts Equation (3) to the groups $\mathbb{Z}_{2}^{\operatorname{Hom}\left(K_{2}, H\right)^{2}}$ and $\mathbb{Z}_{2}^{\operatorname{Hom}\left(K_{2}, S^{m}(H)\right)^{\underline{2}}}$ for all $m \geq 1$. Recall that $g^{+}: \operatorname{Hom}\left(K_{2}, C_{n}\right) \rightarrow \operatorname{Hom}\left(K_{2}, H\right)$ induces $\widehat{g^{+}}$: $\mathbb{Z}_{2}^{\operatorname{Hom}\left(K_{2}, C_{n}\right)^{\underline{2}}} \rightarrow \mathbb{Z}_{2}^{\operatorname{Hom}\left(K_{2}, H\right)^{2}} ; g^{+}$also induces $S^{m}\left(g^{+}\right): S^{m}\left(\operatorname{Hom}\left(K_{2}, C_{n}\right)\right) \rightarrow$ $S^{m}\left(\operatorname{Hom}\left(K_{2}, H\right)\right)$ for all $m \geq 1$, which in turn induce $\widehat{S^{m}\left(g^{+}\right)}: \mathbb{Z}_{2}^{S^{m}}\left(\operatorname{Hom}\left(K_{2}, C_{n}\right)\right)^{2} \rightarrow$ $\mathbb{Z}_{2}^{S^{m}\left(\operatorname{Hom}\left(K_{2}, H\right)\right)^{2}}$.

\section{Lemma 7 .}

$$
\widehat{g^{+}}\left(1_{\operatorname{Hom}\left(K_{2}, C_{n}\right)}^{<}\right)=\sum_{j=1}^{k} 1_{\rho_{j}^{+}}^{<}
$$

hence

$$
\widehat{S^{m}(g)}\left(1_{S^{m}\left(\operatorname{Hom}\left(K_{2}, C_{n}\right)\right)}^{<}\right)=\sum_{j=1}^{k} 1_{S^{m}\left(\rho_{j}^{+}\right)}^{<}
$$

for all $m \geq 1$.

Proof. Let $O_{g}$ be the set of arcs of $H$ which appear an odd number of times as $(g(i), g(i+1))$ or $(g(i+1), g(i))$ for some $i \in \mathbb{Z}_{n}$. Thus in $\mathcal{F}_{\mathcal{V}_{2}}(A(H))$, we have $\sigma_{2}(g)=\sum_{(u, v) \in O_{g}}(u, v)$. By Equation 3, $O_{g}$ coincides with the set of arcs which appear an odd number of times as terms in $\rho_{1}, \ldots, \rho_{k}$.

Now for $(u, v) \in A(H)$, let $V(u, v)$ be the subposet

$$
\left(N_{H}(v),\{v\}\right)>(\{u\},\{v\})<\left(\{u\}, N_{H}(u)\right)
$$

of $\operatorname{Hom}\left(K_{2}, H\right)$. Then

$$
\widehat{g^{+}}\left(1_{\mathrm{Hom}\left(K_{2}, C_{n}\right)}^{<}\right)=\sum_{(u, v) \in O_{g}} 1_{V(u, v)}^{<}
$$

and similarly for all

$$
\widehat{S^{m}(g)}\left(1_{S^{m}\left(\operatorname{Hom}\left(K_{2}, C\right)\right)}^{<}\right)=\sum_{(u, v) \in O_{g}} 1_{S^{m}}^{<}(V(u, v))
$$

for all $m \geq 1$. Equation 3 then implies that

$$
\sum_{(u, v) \in O_{g}} 1_{V(u, v)}^{<}=\sum_{j=1}^{k} 1_{\rho_{j}^{+}}^{<}
$$

and

$$
\sum_{(u, v) \in O_{g}} 1_{S^{m}(V(u, v))}^{<}=\sum_{j=1}^{k} 1_{S^{m}\left(\rho_{j}^{+}\right)}^{<}
$$

for all $m \geq 1$.

We will next see that the conclusion

$$
\widehat{S^{m}(g)}\left(1_{S^{m}\left(\operatorname{Hom}\left(K_{2}, C\right)\right)}^{<}\right)=\sum_{j=1}^{k} 1_{S^{m}\left(\rho_{j}^{+}\right)}^{<}
$$


of Lemma 7 is incompatible with the conclusion

$$
\widehat{S^{m}(g)}\left(1_{S^{m}\left(\operatorname{Hom}\left(K_{2}, C\right)\right)}^{<}\right)=1_{Q_{1}}^{<}
$$

of Lemma 6 .

4.4. Domination and dismantlability. In a poset $Q$, an element $p$ is said to be dominated by an element $q$ if $p$ is comparable to $q$ and every element comparable to $p$ is comparable in the same way to $q$. $Q$ is said to be dismantlable if it can be reduced to a single point by recursively removing dominated elements.
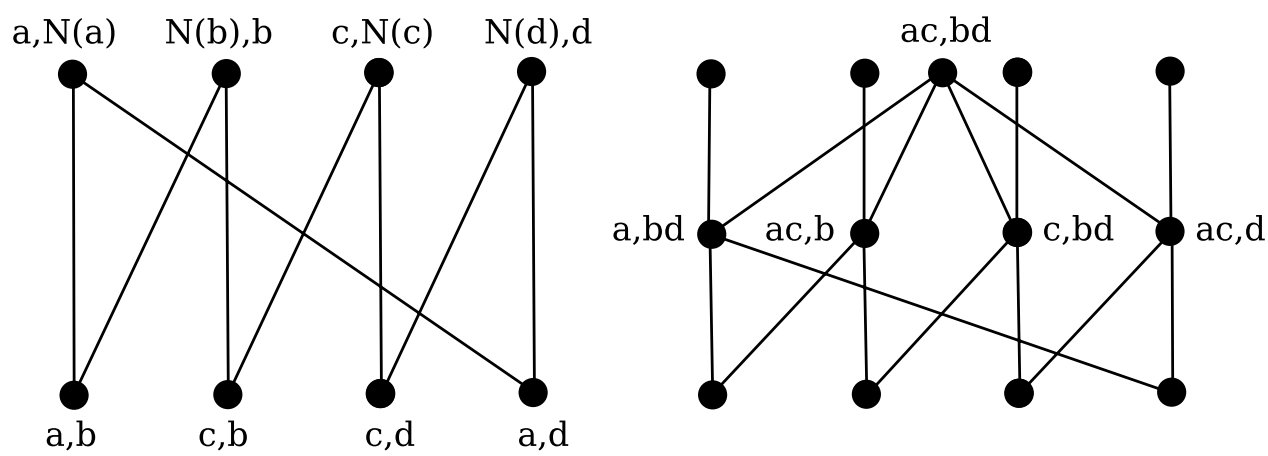

Figure $3 . \rho^{+}$and $D\left(\rho^{+}\right)$

For instance, consider the subposet $D\left(\rho^{+}\right)$of $\operatorname{Hom}\left(K_{2}, H\right)$ obtained by adding the elements $(\{a\},\{b, d\}),(\{a, c\},\{b\}),(\{c\},\{b, d\}),(\{a, c\},\{d\})$ and $(\{a, c\},\{b, d\})$ to $\rho^{+}$. (See Figure 3.) In $D\left(\rho^{+}\right),\left(\{a\}, N_{H}(a)\right),\left(N_{H}(b),\{b\}\right),\left(\{c\}, N_{H}(c)\right)$ and $\left(N_{H}(d),\{d\}\right)$ are dominated respectively by $(\{a\},\{b, d\}),(\{a, c\},\{b\}),(\{c\},\{b, d\})$ and $(\{a, c\},\{d\})$. Removing these dominated elements leaves $(\{a, c\},\{b, d\})$ as the unique maximum. We then have $(\{a\},\{b, d\}),(\{a, c\},\{b\}),(\{c\},\{b, d\})$ and $(\{a, c\},\{d\})$ dominated by $(\{a, c\},\{b, d\})$, and removing these leaves $(\{a\},\{b\})$, $(\{c\},\{b\}),(\{c\},\{b\})$ and $(\{c\},\{d\})$ dominated by $(\{a, c\},\{b, d\})$. Hence $D\left(\rho^{+}\right)$ is dismantlable.

Lemma 8. Let $P$ be a $\mathbb{Z}_{2}$-crown, $Q$ a dismantlable poset and $f: P \rightarrow Q_{1}$ an order-preserving map which factors through $Q$, that is, $f=h \circ g$ where $g: P \rightarrow Q$, $h: Q \rightarrow Q_{1}$ are order-preserving. Then $\hat{f}\left(1_{P}^{<}\right)=0$.

Proof. The result is clear if $Q$ is a single point. Thus we can proceed by induction on the number of elements in $Q$. Let $p$ be dominated by $q$ in $Q$; we will suppose that $p<q$ (the other case being symmetric). Let $r: Q \rightarrow Q$ be the retraction which maps $p$ to $q$ and fixes everything else. Then $f^{\prime}=h \circ r \circ g: P \rightarrow Q_{1}$ factors through the dismantlable poset $r(Q)$ which has one element less than $Q$, so by the induction hypothesis, $\hat{f}^{\prime}\left(1_{P}^{<}\right)=0$. Thus if $f=f^{\prime}$, then $\hat{f}\left(1_{P}^{<}\right)=0$. We can therefore suppose that $f \neq f^{\prime}$. This means that $h(p) \neq h(q)$. We will suppose without loss of generality that $h(p)=0$ and $h(q)=1$.

Let $g^{\prime}: P \rightarrow Q_{1}$ be the map obtained from $g$ by changing the image of every maximal element $x$ such that $g(x)=p$ from $p$ to $q$. For each such $x$, there are two minimal elements $y, z$ of $P$ which are below $x$. We then have $f(y), f(z) \leq f(x)=0$, 
so that $f(y)=f(z)=0$. Therefore the two compararabilities $(y \leq x),(z \leq x)$ are mapped to $(0 \leq 0)$ by $\hat{f}$ and to $(0 \leq 1)$ by $\widehat{h \circ g^{\prime}}$. Thus $\widehat{h \circ g^{\prime}}\left(1_{P}^{<}\right)=\hat{f}\left(1_{P}^{<}\right)$.

Now $r \circ g$ is obtained from $g^{\prime}$ by changing the image of every minimal element $x$ such that $g^{\prime}(x)=p$ from 0 to 1 . For each such $x$, there are two maximal elements $y, z$ of $P$ which are above $x$. Now since $q$ dominates $p$, we have $g^{\prime}(y), g^{\prime}(z) \geq q$ hence $h \circ g^{\prime}(y)=h \circ g^{\prime}(z)=1$. Therefore the two compararabilities $(x \leq y)$, $(x \leq z)$ are mapped to $(0 \leq 1)$ by $\widehat{h \circ g^{\prime}}$ and to $(1 \leq 1)$ by $\widehat{h \circ r \circ g}=\widehat{f^{\prime}}$. Thus $\widehat{f}^{\prime}\left(1_{P}^{<}\right)=\widehat{h \circ g^{\prime}}\left(1_{P}^{<}\right)=\hat{f}\left(1_{P}^{<}\right)$. Therefore $\widehat{f}^{\prime}\left(1_{P}^{<}\right)=0$ implies $\hat{f}\left(1_{P}^{<}\right)=0$.

Lemma 9. If $Q$ is a dismantlable poset, then for any $m, S^{m}(Q)$ is dismantlable.

Proof. It suffices to show that if $Q$ is dismantlable, then $S(Q)$ is dismantlable. We will again proceed by induction on the number of elements in $Q$, the result being clear if $Q$ is a single point. Let $p$ be dominated by $q$ in $Q$. We will show that $S(Q)$ dismantles to $S(Q \backslash\{p\})$. The elements of $S(Q)$ are chains in $Q$, and since $q$ dominates $p$, for every element $C$ of $S(Q)$ containing $p, C \cup\{q\}$ is an element of $S(Q)$. Let $m$ be the number of elements of $S(Q)$ which contain $p$ but not $q$. We construct a sequence $S(Q)=R_{0}, R_{1}, \ldots, R_{m}$ of subposets of $S(Q)$, where $R_{i}$ is obtained from $R_{i-1}$ by removing a maximal element $C_{i}$ of $R_{i-1}$ which contains $p$ but not $q$. Since $C_{i}$ is dominated by $C_{i} \cup\{q\}$ in $R_{i-1}$, the sequence is a dismantling of $S(Q)$ to its subposet $R_{m}$ which consists of all the elements which contain $q$ whenever they contain $p$. Let $R_{m}, R_{m+1}, \ldots, R_{2 m}$ be a sequence of subposets of $R_{m}$, where $R_{i}$ is obtained from $R_{i-1}$ by removing a minimal element $C_{i}$ of $R_{i-1}$ which contains $p$. Since $C_{i}$ is dominated by $C_{i} \backslash\{p\}$ in $R_{i-1}$, the sequence is a dismantling of $R_{m}$ to its subposet $R_{2 m}=S(Q \backslash\{p\})$. Thus $S(Q)$ dismantles to $S(Q \backslash\{p\})$. Therefore if $S(Q \backslash\{p\})$ is dismantlable, then so is $S(Q)$.

Proof of Theorem Q Suppose that for some odd $n$ there exists a homomorphism $f: C_{n} \rightarrow H$ such that $\sigma_{2}(g)=0$. Then there exists a sequence $\rho_{1}, \ldots, \rho_{k}$ of generators of $\theta$ such that $\sigma_{2}(f)=\sum_{j=1}^{k} \rho_{j}$ in $\mathcal{F}_{\mathcal{V}_{2}}(A(H))$. By Lemma 7 , we then have

$$
\widehat{S^{m}\left(f^{+}\right)}\left(1_{S^{m}\left(\operatorname{Hom}\left(K_{2}, C_{n}\right)\right)}^{<}\right)=\sum_{j=1}^{k} 1_{S^{m}\left(\rho_{j}^{+}\right)}^{<}
$$

for all $m \geq 0$. Now for $j=1, \ldots, k, \rho_{j}^{+}$is contained in $D\left(\rho_{j}^{+}\right)$which is dismantlable, hence $S^{m}\left(D\left(\rho_{j}^{+}\right)\right)$is dismantlable for all $m$ by Lemma 9. Hence by Lemma 9, for any order-preserving map $g: S^{m}\left(\operatorname{Hom}\left(K_{2}, H\right)\right) \rightarrow Q_{1}$, we have $\hat{g}\left(1_{S^{m}\left(\rho_{j}^{+}\right)}^{<}\right)=0$ for $j=1, \ldots, k$. Therefore

$$
\left.g \circ \widehat{S^{m}\left(f^{+}\right.}\right)\left(1_{S^{m}\left(\operatorname{Hom}\left(K_{2}, C_{n}\right)\right)}^{<}\right)=\sum_{j=1}^{k} \hat{g}\left(1_{S^{m}\left(\rho_{j}^{+}\right)}^{<}\right)=0 .
$$

By Lemma 6, $g \circ S^{m}\left(f^{+}\right): S^{m}\left(\operatorname{Hom}\left(K_{2}, C_{n}\right)\right) \rightarrow Q_{1}$ cannot be a $\mathbb{Z}_{2}$-map. Since $S^{m}\left(f^{+}\right): S^{m}\left(\operatorname{Hom}\left(K_{2}, C_{n}\right)\right) \rightarrow S^{m}\left(\operatorname{Hom}\left(K_{2}, H\right)\right)$ is a $\mathbb{Z}_{2}$-map, we conclude that there does not exist a $\mathbb{Z}_{2}$-map $g: S^{m}\left(\operatorname{Hom}\left(K_{2}, H\right)\right) \rightarrow Q_{1}$. Therefore $\operatorname{ind}\left(\operatorname{Hom}\left(K_{2}, H\right)\right) \geq$ 2.

\section{REFERENCES}

[1] M. Čadek, M. Krćál, L. Vokřínek, Algorithmic solvability of the lifting-extension problem (2014), 52 pages MS. 
[2] M. Čadek, M. Krćál, J. Matoušek, F Sergeraert, L. Vokřínek, U. Wagner, Computing all maps into a sphere, J. ACM 61 (2014) no. 3, art. 17, 44 pp.

[3] P. Csorba, On the simple $\mathbb{Z}_{2}$-homotopy types of graph complexes and their simple $\mathbb{Z}_{2}$ universality, Canad. Math. Bull. 51 (2008), 535-544.

[4] J. Matoušek, Using the Borsuk-Ulam theorem, Lectures on topological methods in combinatorics and geometry, Written in cooperation with Anders Björner and Günter M. Ziegler, Universitext, Springer-Verlag, Berlin, 2003. xii+196 pp.

[5] W. Imrich, S. Klavžar, Product graphs. Structure and recognition, Wiley-Interscience Series in Discrete Mathematics and Optimization, Wiley-Interscience, New York, 2000.

[6] J, Matoušek, G. Ziegler, Topological lower bounds for the chromatic number: a hierarchy, Jahresber. Deutsch. Math.-Verein. 106 (2004), 71-90.

[7] G. Simons, C. Tardif, D. Wehlau, Generalised Mycielski graphs and bounds on chromatic numbers, 14 pages MS., 2014.

[8] S. Zimmerman, Topological and Algebraic Lower Bounds on the Chromatic Number of Graphs, M. Sc. Thesis, Queen's University, 2014.

Royal Military College of Canada, PO Box 17000 Stn Forces, Kingston, ON, Canada, K7K 7B4 